\title{
Too Close for Comfort: Creating Two TEM Samples From Areas Separated By 270 Nanometers
}

\author{
Nathan Wang ${ }^{1 *}$ and George Perreault ${ }^{1}$ \\ 1. Maxim Integrated, Failure Analysis, San Jose, CA, USA. \\ * Corresponding author: Naiyi.Wang@maximintegrated.com
}

STEM/TEM is the ultimate and generally last technique used in semiconductor failure analysis as the highest resolution images can only be obtained with thin samples and high-energy electron beams [1]. While some of us remember using standalone ion millers, modern techniques rely almost exclusively on FIB tools for TEM sample creation. Because conventional FIB techniques typically require a $20 \mu \mathrm{m} \mathrm{x}$ $20 \mu \mathrm{m}$ sample area, with shrinking sample geometries, a single TEM sample can consume the entire suspected failure site. In response, this technique was developed to obtain multiple laminae from locations separated by less than $500 \mathrm{~nm}$.

To demonstrate this technique, a specimen with two possible failure sites separated by $270 \mathrm{~nm}$ was selected and a lift-out area that encompasses both locations was defined, Figure 1. The area was coated with platinum to help protect the surface, trenches were cut on three sides, the Omniprobe was attached, and the region of interest was lifted out of the failing device and affixed to a TEM grid, Figure 2. To minimize future cutting depth and to help address the taper caused by ion beam diameter [2], the specimen was rotated $90^{\circ}$ and the top and bottom surfaces were milled off, Figure 3 . Because multiple IC layers are needed for defect identification and interpretation, the minimum specimen thickness at this step is approximately 2-3 microns.

The specimen was tilted $-90^{\circ}$ and the two sample sites were located. Notches were made on either side of the samples to minimize the length of future cuts. The Omniprobe was reattached to the free end of the specimen. With the probe properly attached, a FIB cut was made to separate Sample 1 and 2; the smallest ion beam, (9 pA), was selected for this sample delineating final cut to maximize beam resolution [3] and minimize the kerf. Both samples were then thinned, using the FIB ion beam, to prepare the suspect contacts for a TEM investigation, Figure 5.

Results showed that, with some creativity and iterative application of standard FIB sample preparation techniques, it is possible to produce multiple TEM samples from very closely spaced adjacent regions. With older wafer fabrication technologies, good failure analysis results were possible by milling through one location, investigating, and then milling to the next. For modern process nodes, with nanometersize defects and integrated circuit features much less than 0.1 micron, TEM is nearly required at all suspected failure sites. The techniques outlined in this review are, therefore, important for most current investigations, and they will become standard for failure analysis TEM laminae creation as process geometries continue to shrink.

With patience and ingenuity, it is possible to create more than two TEM samples from a single lift-out region. Ion beam-induced specimen taper [2], though, limits the distance between TEM samples to close to the approximately $270 \mathrm{~nm}$ used in this example investigation. 
References:

[1] N Wang and S Li, Electronic Devices Failure Analysis 10 (2008), pp.12-16.

[2] T Fujii et al, in "Focused Ion Beam Systems", ed. Nan Yao, (Cambridge, New York) pp. 382-383.

[3] CA Volkert and AM Minor, MRS Bulletin, 32 (2007), pp. 389-399.

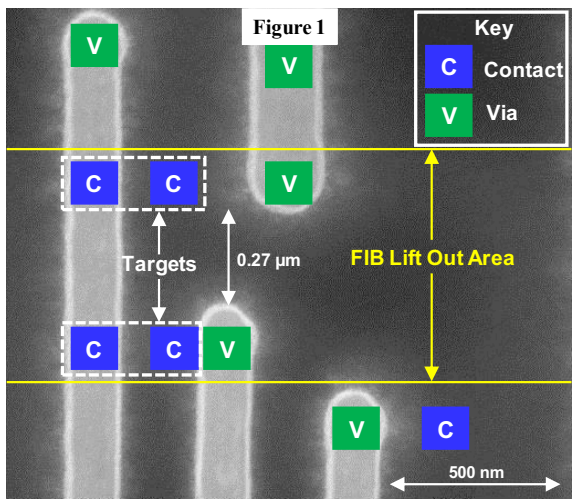

Figure 1. To demonstrate this technique, a specimen with two sites separated by $270 \mathrm{~nm}$ was selected.

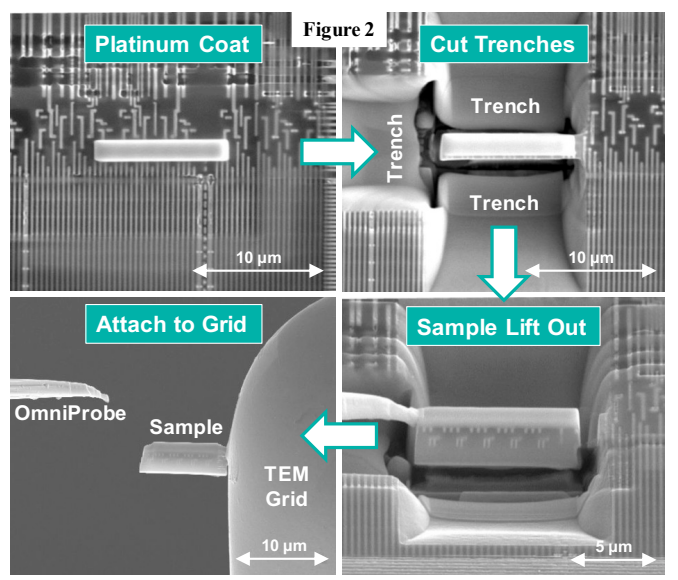

Figure 2. The area was platinum-coated, trenches were cut, the Omniprobe was attached, and the sample was affixed to a TEM grid.

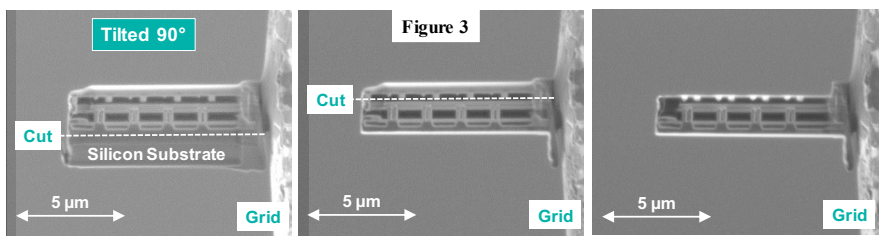

Figure 3. To minimize future cutting, the specimen was tilted $90^{\circ}$ and the top and bottom surfaces were milled off.

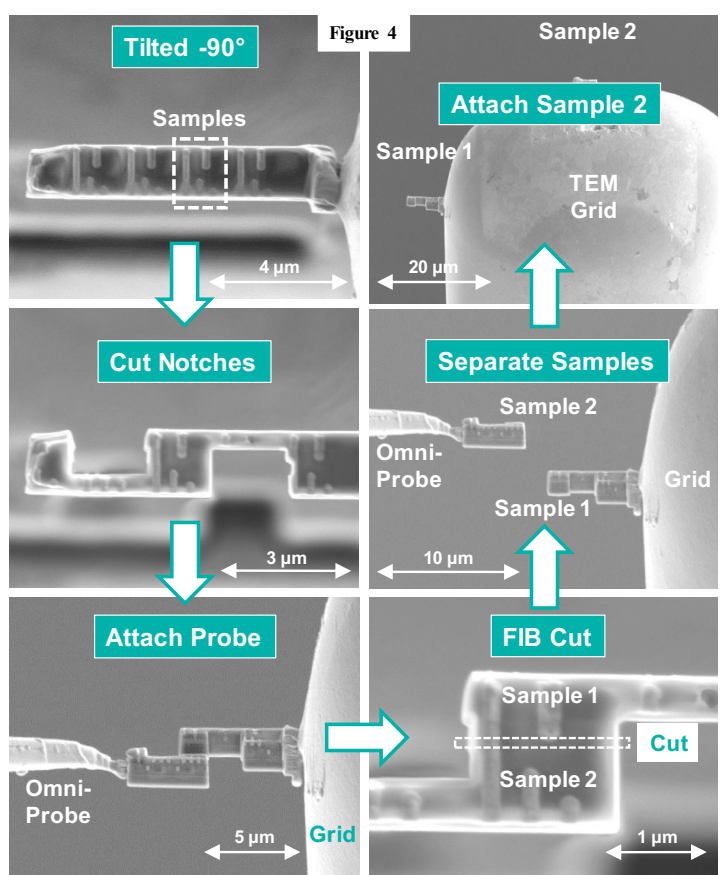

Figure 4. Notches were cut on either side of the samples, the Omniprobe was attached, a FIB cut was made to separate the samples, and Sample 2 was mounted to the TEM grid.

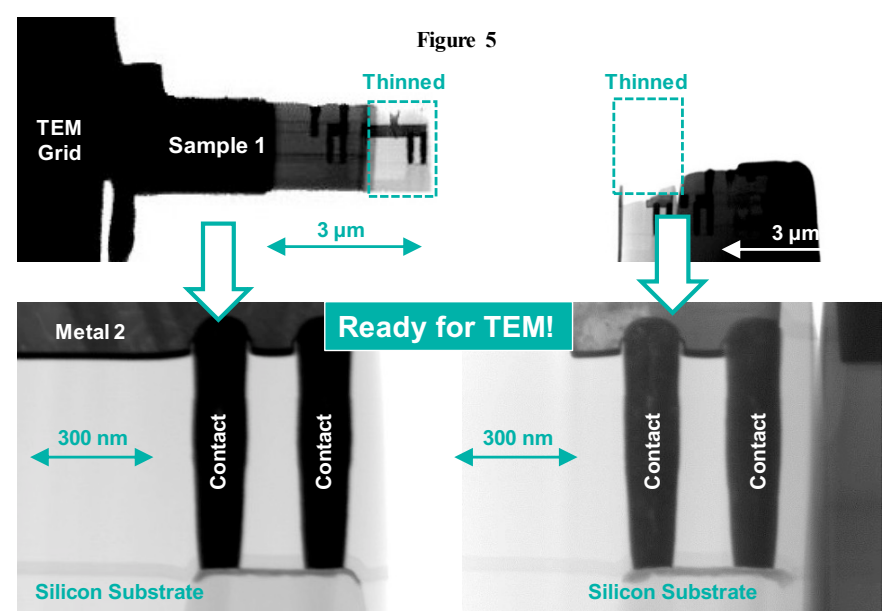

Figure 5. Both samples were thinned to prepare the suspect contacts for a TEM investigation. 